\title{
Economía e información circular en el sector agroalimentario argentino: estudio exploratorio desde la teoría institucional
}

\section{Economy and information circular information in argentinian agrifood sector: an exploratory research from an institutional approach}

\author{
María Laura Rabasedas Catalán ${ }^{1}$, Jose Mariano Moneva Abadía ${ }^{2}$
}

\begin{abstract}
RESUMEN
El impacto ambiental del sector agroalimentario es crítico a nivel mundial dados los altos niveles de contaminación, pérdida de recursos y de energía que le son atribuidos. Así, el desarrollo de la economía circular en el sector aparece como una oportunidad y un desafío. Para esto, la generación de información útil para la toma de decisiones circulares es clave, siendo los reportes de sostenibilidad una importante vía para lograrlo.

En este contexto, la presente investigación busca evaluar el grado de internalización de la economía circular en empresas agroalimentarias argentinas, incluyendo la información circular que se divulga y si existen factores que lo influencian, desde la teoría institucional.

Se trata de un estudio descriptivo y exploratorio de corte mixto. Para llevarlo adelante se analizaron 210 respuestas recibidas a una encuesta diseñada ad hoc, mediante estadística descriptiva y test de diferencias de medias para contrastar las hipótesis.

Los resultados generales muestran que los niveles de internalización son bajos tanto en la estrategia, prácticas como en la información circular que se divulga. Las empresas agroalimentarias argentinas se ven afectadas por factores institucionales para implementar la economía circular. Futuras investigaciones deben concentrarse en entender las motivaciones y barreras que deben superarse para lograr una verdadera adopción de la economía circular en el sector.
\end{abstract}

Palabras Clave: agroalimentos, economía circular, información y contabilidad circular.

Recepción: 19/10/2021 Aprobación: 19/11/2021

\footnotetext{
1 Universidad Nacional del Litoral, Facultad de Ciencias económicas, Santa Fe, Argentina, mlrabasedas@fce.unl.edu.ar 2 Universidad de Zaragoza, Facultad de Economía y Empresas. Zaragoza, España, jmmoneva@unizar.es
} 


\begin{abstract}
The environmental impact of the agri-food sector is critical given the high levels of pollution and food loss that are generated. The development of the circular economy in the sector appears as both an opportunity and as a challenge. To do so, it is necessary to generate useful information for circular decisions, with sustainability reports being an important instrument.

In this context, this research seeks to evaluate the degree of internalization of the circular economy in Argentinian agri-food companies, including circular information disclosed and factors that influence, from an institutional theory perspective.

An ad hoc questionnaire survey was sent to Argentinian agri-food companies, of which 210 responded. Data analysis was performed using descriptive statistics and media difference to test the hypothesis.

Results show that levels of internalization are low in all the categories: strategy, practices and circular information disclosure. Also, Argentinian agri-food companies are affected by institutional factors. Future research could focus on understanding the motivations and barriers that must be overcome to achieve true adoption of the circular economy in the sector.
\end{abstract}

Keywords: agrifood, circular economy, circular information and accounting.

\title{
INTRODUCCIÓN
}

La economía circular (EC) es un modelo que ha ganado popularidad en los últimos años, (Geissdoerfer et al., 2017; Ghisellini et al., 2016). Opuesto a la idea de la economía lineal, busca que los recursos permanezcan en uso y valor durante el mayor tiempo posible, evitando desde el origen la generación de residuos y desperdicios (Ellen Macarthur Foundation, 2015; Kirchherr et al., 2017). Si bien la EC es imperante en todos los sectores económicos, el sector agroalimentario destaca por la trascendencia de los bienes y servicios que provee y por los altos niveles de contaminación, pérdida de alimentos, recursos y energía que le son atribuidos (Esposito et al., 2020). Esta situación, sumada a la creciente demanda de alimentos a nivel mundial (Salimi, 2021), constituyen grandes desafíos para el desarrollo de la EC en el sector (Muscio \& Sisto, 2020).

En términos generales, la EC está siendo ampliamente estudiada. En el caso específico del sector agroalimentario, las investigaciones discuten diversos aspectos: modelos de negocios (Fattahi et al., 2013; Nasution et al., 2020); motivaciones y barreras (Farooque et al., 2019); manejo de residuos y desecho de alimentos (Duque-Acevedo et al., 2020); packaging (Kleine Jäger \& Piscicelli, 2021). La mayor parte de estos estudios centran su análisis en el contexto de países desarrollados, principalmente China y Europa (Reike et al., 2018; Scarpellini, Valero-Gil, et al., 2020). Latinoamérica, por su parte, detenta progresos incipientes y Argentina en particular, cuenta con avances que se ubican en etapas iniciales (Rodríguez et al., 2018) aunque en crecimiento. El tema, paulatinamente, se está institucionalizando, siendo posible encontrar dentro de organismos públicos áreas dedicadas a la EC. Sin embargo, las investigaciones que abordan la implementación en Argentina son insuficientes, dejando una brecha que debe ser cubierta. 
Como puede inferirse, la implementación concreta de la EC en el sector es un tema en discusión, que requiere la generación de información útil y oportuna para la toma de decisiones circulares tanto interna como externa (Fortunati et al., 2020; Stewart \& Niero, 2018a). En esto, la contabilidad no financiera, a través de los reportes de sostenibilidad (RS), cumple un importante rol, siendo relativamente escasos y recientes los estudios que se ocupan del tema desde esta perspectiva (Gunarathne et al., 2021; Scarpellini et al., 2020).

En el contexto descrito, el objetivo de este artículo es evaluar el grado de implantación de la EC en las organizaciones agroalimentarias de Argentina, respondiendo a tres preguntas de investigación: 1. ¿Cuál es el grado de implantación de la EC en la estrategia organizacional de empresas agroalimentarias de Argentina, así como las prácticas de EC llevadas a cabo?; 2. ¿Se genera y publica información interna y externa sobre circularidad?; y 3. ¿Qué características organizacionales influencian el grado de implantación de la EC?.

Lo que resta del artículo se estructura de la siguiente manera. La próxima sección desarrolla las hipótesis y luego se explica la metodología de investigación aplicada. En el apartado siguiente, se presentan los resultados obtenidos y, por último, la discusión de resultados y conclusiones.

\section{Desarrollo de Hipótesis}

Tanto la pregunta de investigación 1 como la 2, se evaluarán con análisis descriptivo. Para responder la pregunta de investigación 3, siguiendo a Dagiliene et al., (2020), se utilizará el enfoque de la teoría institucional (Powell \& DiMaggio, 1991). El desarrollo de hipótesis se expone a continuación:

De acuerdo con Gray et al., (2009), la nacionalidad de una empresa matriz afecta al comportamiento y al alcance y contenido de las revelaciones ambientales de sus subsidiarias. En este sentido, la cultura y normativas ambientales del país de la matriz generalmente se reflejan en el comportamiento de sus filiales en relación con la protección del medio ambiente (Moneva \& Llena, 2000). Particularmente, Argentina es un país en el que la EC se encuentra recién en sus inicios, por lo que la influencia de empresas matrices de otros países que cuentan con mayor trayectoria en el tema, podría afectar el nivel de adopción de la EC y su divulgación.

Hipótesis 1: Las organizaciones con controlador extranjero tendrán más internalizada la EC tanto en estrategia, prácticas, así como en la divulgación de información de EC.

Por otro lado, las empresas que cotizan en bolsa, son organizaciones con mayor exposición pública, que están bajo un cercano control de los inversores y de las instituciones que regulan el mercado de capitales, por lo que deben adoptar las regulaciones ambientales, divulgar los resultados y ser innovadoras en este sentido, respondiendo por tanto a presiones institucionales. Según estudios realizados en China, las entidades que cotizan en bolsa tienden a divulgar más información contable de EC (Wang et al., 2014), y esto hace que los inversores obtengan una comprensión más acabada de las actividades circulares de la empresa (Dagiliene et al., 2020) y llevar a las organizaciones, por tanto, a realizar negocios más circulares. 
Hipótesis 2: Las organizaciones cotizadas tendrán más internalizada la EC tanto en su estrategia, prácticas, así como en la divulgación de información sobre EC

La adopción de estándares para divulgación información no financiera mediante RS, podría llevar a las empresas a incorporar la circularidad en sus acciones (Scarpellini et al., 2020) y generar mayor divulgación relacionada con la EC, siendo afectadas así por factores normativos relacionados con el colectivo profesional (Powell \& DiMaggio, 1991).

Hipótesis 3: Las organizaciones que publican un RS tendrán más internalizada la EC tanto en su estrategia, prácticas, así como en la divulgación de información sobre EC

El isomorfismo mimético surge cuando las entidades tienden a seguir reglas y rutinas de otras organizaciones más exitosas ante contextos inciertos. En consecuencia, asumimos que las empresas líderes de un subsector determinado, que lleven adelante acciones de EC e informen sobre las mismas, influirán positivamente en otras empresas del mismo subsector (Dagiliene et al., 2020).

Hipótesis 4: Las organizaciones en subsectores con líderes tendrán más internalizada la EC tanto en su estrategia, prácticas, así como en la divulgación de información sobre EC.

\section{METODOLOGÍA, MATERIALES Y MÉTODOS}

\section{Diseño y prueba de instrumento}

Para abordar el objetivo del estudio se elaboró un cuestionario ad hoc tipo encuesta, que se compuso de 5 secciones (ver Anexo): Aspectos generales, Estrategia y cultura (6 ítems); Prácticas de EC dividida en 4 áreas: Abastecimiento (5 ítems), Producción (7 ítems), comercialización y distribución (9 ítems) y destino final (5 ítems); Información circular (7 ítems). Las preguntas incluidas dentro de cada sección fueron desarrolladas en base a estudios anteriores relacionados con la implantación de la EC; con el sector agroalimentario y con información de sostenibilidad y circular (Bhimani et al., 2016; Ellen Macarthur Foundation, 2017; Laboratorio de Ecoinnovación, 2017; ONU FAO, 2020; PACE, 2021; Salimi, 2021; Stewart \& Niero, 2018b; Thorne et al., 2014; Verbeek, 2016; Windolph et al., 2014; Zhu et al., 2010). En la mayoría de las preguntas se aplicó una escala de Likert de 4 puntos. El cuestionario fue enviado para su validación a cuatro especialistas en el área de la EC e información circular (en adelante, IC) para determinar la idoneidad de cada elemento de medición y evaluar su claridad.

La encuesta fue dirigida a 2500 organizaciones del sector agroalimentario vinculadas a diferentes cámaras empresariales. El período de envío y recepción de respuestas fue de abril a julio 2021. Se recibieron 210 respuestas completas, lo que implica un ratio de respuestas de 8,4\%, similar al obtenido en otros estudios de la temática (Mura et al., 2020; Sumter et al., 2021). 
Las empresas fueron clasificadas en función a cuatro variables: Sector, utilizando para esto una adaptación de Global Industry Classification Standard (MSCI, 2020), dado su alcance internacional; Cotizada, variable dicotómica que indica si la entidad cotiza en algún mercado de valores; Tipo de controlador, nacional o extranjero, en función al origen del capital mayoritario; y RS, variable también dicotómica que refleja la publicación o no de un RS.

En función al objetivo del presente artículo, se trabajará con tres constructos: Estrategia y cultura de EC; Prácticas de EC e IC. La información fue analizada utilizando estadística descriptiva y no paramétrica a través del test de Kruskal Wallis y correlaciones de Spearman.

Para testear la confiabilidad de la encuesta en cuanto a su capacidad de medición se utilizaron, en una primera etapa, el alfa de Cronbach y las correlaciones ítem-total corregidas y en una segunda etapa se realizó un análisis factorial exploratorio utilizando la técnica de componentes principales (ACP) con rotación varimax (Hair et al., 2005) (ver ¡Error! La autoreferencia al marcador no es válida.).

\section{Tabla 1}

Testeo de consistencia, fiabilidad y unidimensionalidad.

\begin{tabular}{lcccccc}
\hline & $\mathbf{N}$ & $\begin{array}{c}\text { Alfa de } \\
\text { Cronbach }\end{array}$ & $\begin{array}{c}\text { Correlación } \\
\text { item-total }\end{array}$ & $\mathbf{N}$ & Cargas ACP & N final \\
\hline Estrategia y cultura de EC & 6 & 0,910 & $0,54-0,84$ & 6 & $0,639-0,905$ & 6 \\
\hline Prácticas de EC & 26 & 0,914 & $0,38-0,64$ & $24^{*}$ & $0.599-0.807$ & $22^{* *}$ \\
\hline Información circular & 7 & 0,923 & $0,58-0,92$ & 7 & $0,693-0,942$ & 7 \\
\hline
\end{tabular}

Nota: Se muestran los resultados del test alfa de Cronbach y las correlaciones item-total para los constructos. Los niveles alcanzados sugieren que las variables se ajustan razonablemente bien a los datos.

(*) Se eliminaron 2 ítems dentro de Prácticas de EC, área comercialización por tener una correlación menor a 0.30 (FríasNavarro, 2021; Hair et al., 2005). Así, el N luego de esta etapa pasé de 26 a 24 ítems.

$\left.{ }^{* *}\right)$ Se eliminaron 2 ítems adicionales luego del ACP en la variable "Prácticas de EC", área destino final por cargar sobre un segundo factor.

\section{Descripción de la muestra}

En la Tabla 2 se refleja la representación de los subsectores en la muestra. Respecto a la cotización, $176(84 \%)$ de las empresas no cotizan en bolsa y 34 (16\%) sí lo hacen. Por otro lado, $48(23 \%)$ empresas poseen controlador extranjero y 162 (77\%) nacional. Estos resultados son consistentes, en términos generales, con estadísticas oficiales y estudios previos que analizan la estructura del sector en el país (Palmieri y Pierini, 2018; Ministerio de desarrollo productivo, 2021; CNV, 2021). 


\section{Tabla 2}

Distribución respuesta por subsectores económicos.

\begin{tabular}{|c|c|c|c|c|c|}
\hline Subsectores & Frec & Porcentaje & Subsectores & Frec & Porcentaje \\
\hline 1. Producción agropecuaria & 59 & $28,10 \%$ & $\begin{array}{l}\text { 3. Procesados y ultra } \\
\text { procesados }\end{array}$ & 39 & $18,57 \%$ \\
\hline $\begin{array}{l}\text { 2. Proceso de Productos } \\
\text { primarios }\end{array}$ & 63 & $30,00 \%$ & $\begin{array}{l}\text { 3.1 Aderezos, especias y } \\
\text { extractos }\end{array}$ & 8 & $3,81 \%$ \\
\hline 2.1 Aceites y grasas & 10 & $4,76 \%$ & 3.2 Alimentación animal & 5 & $2,38 \%$ \\
\hline 2.2 Industria cárnica & 17 & $8,10 \%$ & 3.3 Galletas y golosinas & 8 & $3,81 \%$ \\
\hline 2.3 Industria de pescado & 6 & $2,86 \%$ & $\begin{array}{l}\text { 3.4 Panadería y pastas } \\
\text { alimenticias }\end{array}$ & 13 & $6,19 \%$ \\
\hline 2.4 Molineras y almidones & 17 & $8,10 \%$ & 3.5 Otros & 5 & $2,38 \%$ \\
\hline $\begin{array}{l}2.5 \text { Prep. y cons. de frutas y } \\
\text { hortalizas }\end{array}$ & 10 & $4,76 \%$ & 4. Productos lácteos & 17 & $8,10 \%$ \\
\hline \multirow[t]{3}{*}{2.6 Otros } & 3 & $1,43 \%$ & 5. Bebidas & 32 & $15,24 \%$ \\
\hline & & & 5.1 Bebidas alcohólicas & 24 & $11,43 \%$ \\
\hline & & & $\begin{array}{l}\text { 5.2 Bebidas sin alcohol } \\
\text { (excepto leche) }\end{array}$ & 8 & $3,81 \%$ \\
\hline
\end{tabular}

Nota: Representación de los subsectores en la muestra.

\section{RESULTADOS}

Respecto a la estrategia de EC, las medias por ítem (ver Anexo Sección Estrategia de EC) rondan los 2 puntos, lo que implica un puntaje "bajo" a "medio". La desviación típica en todos los casos es mayor o igual a 1, indicando una alta dispersión en las respuestas. Un análisis por organización, permite observar que hay 43 empresas (20\%) que tienen media 1, es decir, nula consideración de la EC en su estrategia, contra 11 organizaciones $(5 \%)$ que tienen media 4, alta internalización. El 60\% de las respuestas, se concentra en medias entre 1 y 2, niveles nulo-bajo y bajo-medio.

En cuanto a las Prácticas de EC por área del proceso, encontramos que las tres más realizadas refieren a gestión de residuos y técnicas de eficiencia en producción, así como packaging para conservación (ver Tabla 3). Las menos realizadas refieren a utilización de biocombustibles para el transporte y el proceso productivo y logística inversa de empaque.

En general, las medias de todos los ítems preguntados en esta sección no superan los 3 puntos, demostrando niveles medios de aplicación, aunque más altos que en el caso de Estrategia. 


\section{Tabla 3}

Top 3 de prácticas de EC más y menos realizadas.

\begin{tabular}{llc}
\hline \multicolumn{1}{c}{ Área } & \multicolumn{1}{c}{ Detalle } & Porcentaje \\
\hline Top 3 de práctica más realizadas & $\begin{array}{l}\text { Los residuos no reutilizables son tratados para disminuir al } \\
\text { máximo los efectos negativos de su disposición final. }\end{array}$ & $84,76 \%$ \\
\hline Destino Final & $\begin{array}{l}\text { Técnicas de producción que reducen al mínimo el empleo de } \\
\text { inputs y servicios }\end{array}$ & $84,29 \%$ \\
Producción & $\begin{array}{l}\text { El packaging empleado mantiene seguros los alimentos } \\
\text { reduciendo deterioros y brindando más tiempo consumo }\end{array}$ & $83,81 \%$ \\
$\begin{array}{lll}\text { Comercialización y } \\
\text { distribución }\end{array}$ & $\begin{array}{l}\text { La energía consumida proviene de fuentes renovables } \\
\text { Top 3 de práctica menos realizadas }\end{array}$ & $42,86 \%$ \\
\hline $\begin{array}{l}\text { Producción } \\
\text { Comer. y distribución }\end{array}$ & $\begin{array}{l}\text { Logística inversa de empaque o cooperación con terceros en } \\
\text { sistema de depósito }\end{array}$ & $34,29 \%$ \\
Comer. y distribución & $\begin{array}{l}\text { Los modos de transporte utilizados son eléctricos o } \\
\text { biocombustibles }\end{array}$ & $26,67 \%$ \\
\hline
\end{tabular}

Fuente: Elaboración propia.

Si analizamos por organización y según el área, las medias más altas (entre 3 y 4) se encuentran en Producción y destino final. Las medias más bajas se ubican en las áreas de comercialización y distribución. En todos los casos, predominan las organizaciones con medias entre 2 y 3 , es decir niveles bajos-medios de aplicación.

En cuanto al análisis de las respuestas en la sección de IC una gran mayoría de las organizaciones (160 - 76\% ) no publica RS o documento similar. De las que sí lo hacen, el 5\% (11) lo publican de forma integrada y 19\% (39) de forma separada, es decir, como un documento individual. Del total de 50 empresas que publican RS, 42\% (21) no incluye la EC, 34\% (17) lo hacen incluido en otros apartados (mayormente el ambiental) y un $24 \%$ (12) en un apartado específico.

Respecto a la IC en sí, las medias generales son bajas, todas menores a 2, lo que las ubica en un nivel Nulo-bajo de la escala (ver Anexo Sección IC), por debajo incluso que Estrategia y Prácticas. Un análisis de frecuencia de respuesta por categoría, permite ver que "1" fue la opción más elegida, es decir, se trata de aspectos no considerados por las organizaciones en general.

Para analizar la vinculación entre los constructos, se realizó un análisis de correlación utilizando la técnica de Rho de Spearman (ver Tabla 4). Los resultados muestran correlaciones positivas significativas en todas las variables. La correlación más fuerte se da entre Estrategia de EC e IC y se puede clasificar como considerable (Hernández \& Fernández, 1998). Prácticas de EC tiene una correlación media con IC y con Estrategia. Un análisis de correlaciones ítem por ítem muestra que las Prácticas de EC tienen correlaciones positivas entre débiles y medias (Hernández \& Fernández, 1998) con las categorías dentro de Estrategias (0.14 a 0.49) y de IC (0.17 a 0.533$)$. 


\section{Tabla 4}

Correlaciones de Spearman.

\begin{tabular}{cccc}
\hline & IC & Estrategia & Prácticas \\
\hline IC & 1,000 & & \\
\hline Estrategia & $0,701^{* * *}$ & 1,000 & \\
\hline Prácticas & $0,586^{* * *}$ & $0,57^{* * *}$ & 1,000 \\
\hline
\end{tabular}

Nota: ${ }^{* * *}$ La correlación es significativa al nivel 0,01 (bilateral).

\section{Contrastación de hipótesis}

Diferencia de medias por controlador: Los resultados indican que existen diferencias significativas entre las medias de las variables, por lo que no es posible rechazar H1 (ver Tabla 5). Las entidades con controlador extranjero presentan medias más altas en los tres casos (ver Figura 1).

\section{Tabla 5}

Resultados diferencia de medias según tipo de controlador.

\begin{tabular}{lllllc}
\hline & Controlador & $\mathrm{n}$ & Media & DE & U de Mann-Whitney \\
\hline \multirow{2}{*}{ Estrategia y cultura EC } & Nacional & 162 & 1,886 & 0,818 & $2213^{* * *}$ \\
& Extranjero & 48 & 2,604 & 0,957 & \\
\hline \multirow{2}{*}{ Prácticas de EC } & Nacional & 162 & 2,189 & 0,525 & $2183,5^{* * *}$ \\
& Extranjero & 48 & 2,611 & 0,612 & $2161^{* * *}$ \\
\multirow{2}{*}{ IC } & Nacional & 162 & 1,608 & 0,765 & \\
\hline
\end{tabular}

Nota: ${ }^{* * *}$ La correlación es significativa al nivel 0,01 (bilateral).

\section{Figura 1}

Medias por categoría según tipo de controlador.

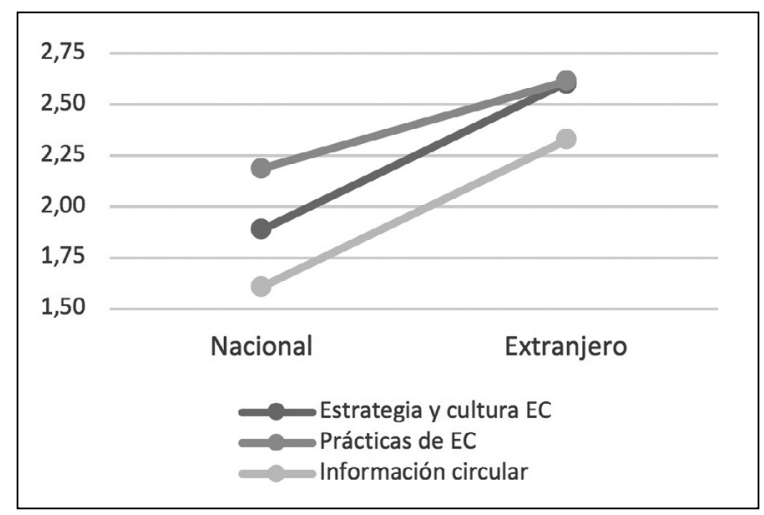

Nota: se muestran las medias por categoría analizadas según tipo de controlador nacional o extranjero. 
Diferencia de medias por cotizada/no cotizada: Los resultados indican que existen diferencias significativas entre las medias de las tres variables (ver ¡Error! No se encuentra el origen de la referencia.). No es posible rechazar $\mathrm{H} 2$ : Las organizaciones cotizadas tienen más internalizada la EC, tanto en su estrategia, prácticas, así como en la divulgación de información sobre EC, en comparación con las no cotizadas (ver Figura 2).

\section{Tabla 6}

Resultados diferencia de medias cotizadas.

\begin{tabular}{lccccc}
\hline & Cotizada & $\mathrm{n}$ & Media & DE & U de Mann-Whitney \\
\hline \multirow{2}{*}{ Estrategia y cultura EC } & $\mathrm{No}$ & 176 & 1,895 & 0,806 & $1376^{* * *}$ \\
& $\mathrm{Si}$ & 34 & 2,853 & 0,953 & \\
\hline \multirow{2}{*}{ Prácticas de EC } & $\mathrm{No}$ & 176 & 2,204 & 0,529 & $1460,5^{* * *}$ \\
\hline \multirow{2}{*}{ IC } & $\mathrm{Si}$ & 34 & 2,706 & 0,610 & $1456^{* * *}$ \\
& $\mathrm{No}$ & 176 & 1,638 & 0,783 & \\
\hline
\end{tabular}

Nota: ${ }^{* *}$ La correlación es significativa al nivel 0,01 (bilateral).

\section{Figura 2}

Medias por categoría según variable cotizada.

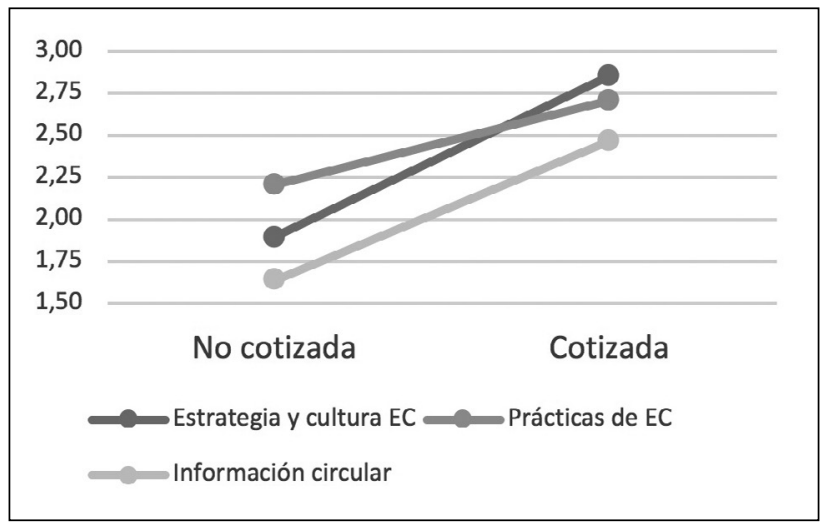

Nota: se muestran las medias por categoría analizadas según la entidad sea cotizada o no cotizada.

Diferencia de medias según publica RS: Para este caso, no es posible rechazar H3 (ver ¡Error! No se encuentra el origen de la referencia.). Las empresas que publican RS tienen más internalizada la EC tanto en su estrategia, prácticas, así como en la divulgación de información sobre EC, en comparación con las que no lo publican (ver Figura 3). 


\section{Tabla 7}

Resultados diferencia de medias RS.

\begin{tabular}{lccccc}
\hline & RS & $\mathrm{n}$ & Media & Desv. Est. & U de Mann-Whitney \\
\hline \multirow{2}{*}{ Estrategia y cultura de EC } & No & 160 & 1,801 & 0,751 & $1524,5^{* * *}$ \\
& $\mathrm{Si}$ & 50 & 2,847 & 0,886 & \\
\hline \multirow{2}{*}{ Prácticas de EC } & No & 160 & 2,141 & 0,513 & $1624^{* * *}$ \\
\hline \multirow{2}{*}{ IC } & $\mathrm{Si}$ & 50 & 2,746 & 0,510 & \\
\hline
\end{tabular}

Nota: ${ }^{* * *}$ La correlación es significativa al nivel 0,01 (bilateral).

\section{Figura 3}

Medias por categoría según variable RS.

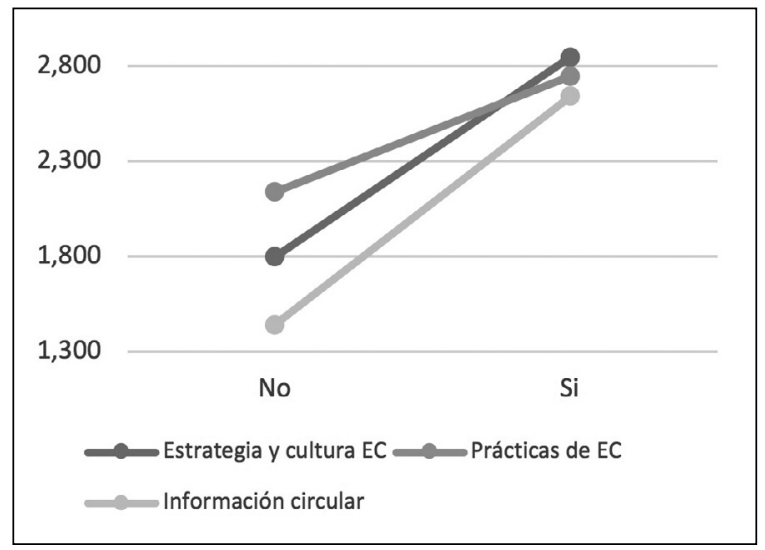

Nota: se muestran las medias por categoría analizadas según la entidad publique o no un RS.

Diferencia de medias por sector: Los resultados indican que existen diferencias significativas entre las medias de las variables Estrategia y Prácticas. No así para el caso de IC (ver Tabla 8). Siguiendo a Dagiliene et al. (2020), para verificar esta hipótesis se normalizó la muestra según el tamaño más pequeño de sub-industria. Como el sector 3.2 (Alimentación animal) incluye 5 organizaciones, se tomaron las 5 empresas de cada sector que presentan mayores medias en cada variable con diferencia significativa: Estrategia y Prácticas de EC. A continuación, se seleccionaron las 10 mejores empresas líderes de la muestra normalizada y se verificó que lo sectores con medias por encima del promedio tengan líderes en la lista. Podemos concluir que en ambos casos la mayoría de sectores con medias superior al promedio tienen líderes (Bebidas, Galletas y golosi- 
nas, Industria de pescado y Otros para Estrategia de EC; y Alimentación animal, Bebidas, Galletas y golosinas, Industria de pescado y Productos Lácteos para Prácticas de EC). Solo Producción Agropecuaria en ambos casos, tuvo media por debajo del promedio y a la vez un líder en el TOP 10. Por otro lado, existen tres sectores que no tienen líderes, pero sí media mayor al promedio: Alimentación animal, Aceites y grasas y Aderezos, especias y extractos para Estrategia de EC y Aceites y grasas, Aderezos, especias y extractos y Otros para Prácticas de EC (Ver Figura 4).

Por lo expuesto, entendemos que no es posible rechazar $\mathrm{H} 4$ en lo que respecta a Estrategia y Prácticas de EC, lo que implica que empresas de subindustrias con líderes identificados tienden, en general, a tener medias más altas que empresas pertenecientes a otras subindustrias.

\section{Tabla 8}

\section{Resultados diferencia de medias por sector.}

\begin{tabular}{|c|c|c|c|c|c|}
\hline & Sectores & $\mathbf{N}$ & Media & DE & Kruskal-Wallis \\
\hline \multirow{6}{*}{ Estrategia y Cultura de EC } & Bebidas sin alcohol & 8 & 2,917 & 0,992 & \multirow[t]{6}{*}{$27,488^{* * *}$} \\
\hline & Alimentación animal & 6 & 2,750 & 0,648 & \\
\hline & Galletas y golosinas & 8 & 2,708 & 1,087 & \\
\hline & Otros & 16 & 2,490 & 1,060 & \\
\hline & Industria del pescado & 6 & 2,306 & 0,846 & \\
\hline & Total & 210 & 2,050 & 0,901 & \\
\hline \multirow{7}{*}{ Prácticas de EC } & Alimentación animal & 6 & 2,808 & 0,603 & \multirow[t]{7}{*}{$30,677^{* * *}$} \\
\hline & Bebidas sin alcohol & 8 & 2,706 & 0,564 & \\
\hline & Galletas y golosinas & 8 & 2,519 & 0,630 & \\
\hline & Aceites y grasas & 10 & 2,518 & 0,532 & \\
\hline & Industria del pescado & 6 & 2,475 & 0,737 & \\
\hline & $\cdots$ & & & & \\
\hline & Total & 210 & 2,285 & 0,572 & \\
\hline \multirow{7}{*}{$\mathrm{IC}$} & Bebidas sin alcohol & 8 & 2,804 & 1,096 & \multirow[t]{7}{*}{18,238} \\
\hline & Alimentación animal & 6 & 2,143 & 1,099 & \\
\hline & Industria del pescado & 6 & 2,095 & 1,131 & \\
\hline & Otros & 16 & 2,089 & 1,041 & \\
\hline & Galletas y golosinas & 8 & 2,071 & 1,122 & \\
\hline & $\cdots$ & & & & \\
\hline & Total & 210 & 1,772 & 0,866 & \\
\hline
\end{tabular}

Nota: Se presentan los 5 sectores con mayores medias.

${ }^{* * *}$ La correlación es significativa al nivel 0,01 (bilateral). 


\section{Figura 4}

Medias por categoría según sector.
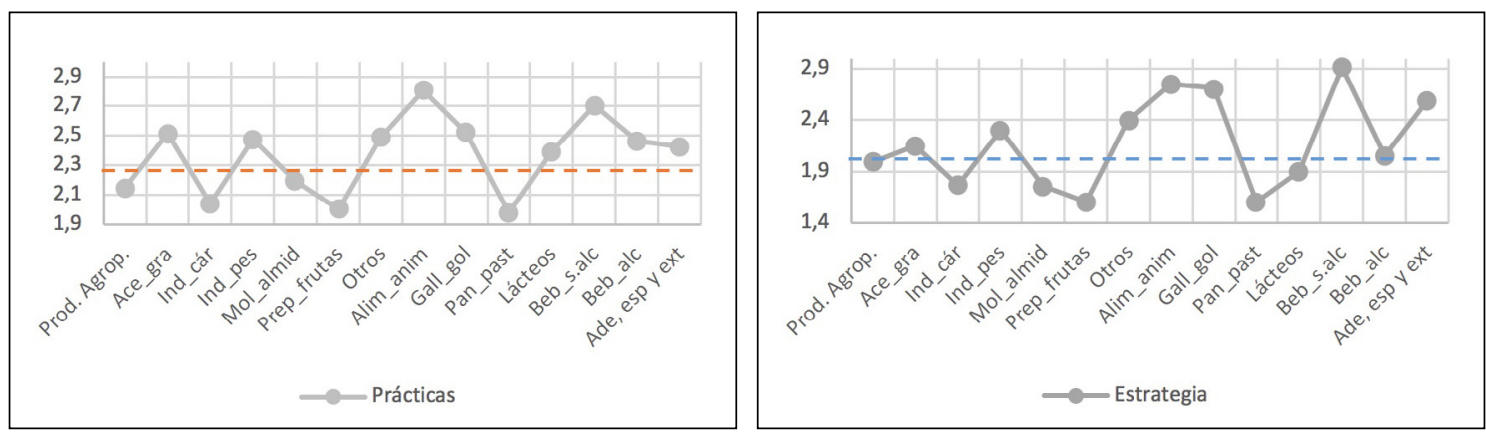

Nota: Se muestran las medias por subsector obtenidas en las variables Prácticas y Estrategia de EC.

\section{DISCUSIÓN y CONCLUSIONES}

El presente estudio buscó explorar cómo las organizaciones del sector agroalimentario argentino han adoptado la EC dentro de su estrategia, en sus prácticas y en la generación de IC. Para cumplir los objetivos, se diseñó y envió una encuesta ad hoc y se aplicaron técnicas de estadística descriptiva y tests no paramétricos para contrastar las hipótesis desarrolladas desde la teoría institucional. Como conclusión general, si bien la EC está creciendo, todavía no ha sido suficientemente adoptada por las organizaciones del sector, ya que tanto los niveles de internalización como los de aplicación de prácticas son relativamente bajos.

Continuando con el análisis, los resultados respecto a la generación de IC, muestran que fue la categoría con menores medias en el total del análisis. Esto no sorprende si consideramos que un $25 \%$ de organizaciones de la muestra publica un RS, siendo consistente con estudios previos que analizan la información de sostenibilidad en el país (Del Barco Zilli et al., 2018; Pastor et al., 2019; Rabasedas, 2018). Un motivo considerable para estos niveles, refiere a la inexistencia de marcos normativos que regulen la emisión de información circular.

En el análisis correlacional pudo verse que las prácticas de EC están relacionadas, en niveles medios, con la estrategia y la IC, es decir que, las organizaciones aplican prácticas de EC (en mayor o menor medida), pero no todas cuentan con una estrategia de EC e IC. Una posible explicación refiere a la baja institucionalización del tema, lo que lleva a no incluirlo explícitamente como parte de la estrategia o en la información que se genera. Esto puede deberse en parte, a una difusa -o nula- percepción sobre el concepto de EC, es decir, que las entidades pueden estar realizando acciones de EC, pero fuera del "paraguas de la EC" (Barreiro-Gen \& Lozano, 2020). Entendemos que el camino para lograr superar esta brecha está en fomentar la educación y capacitación en el tema. Coincidimos con Mura et al. (2020) en que hace falta una comunicación más profunda y eficiente sobre historias de éxito y las oportunidades que ofrece la EC para mejorar la vinculación entre estrategia y acciones concretas (Barreiro-Gen \& Lozano, 2020).

También se encontró que las organizaciones del sector responden a lógicas institucionales. 
Esto implica que en Argentina se requiere una activa y direccionada participación del Estado como ente regulador, tanto en la fijación de normativas como en la aplicación de incentivos que entre otros aspectos la divulgación de información (Liu \& Bai, 2014). Esto no sólo ocurre en Argentina: la necesidad de regulaciones claras, no fragmentadas, disminución de la burocracia y la generación de incentivos, aparecen como los grandes desafíos a superar en diversos estudios de la temática (Dagiliene et al., 2020; Mura et al., 2020).

Para finalizar es importante destacar algunas limitaciones del estudio. Respecto al instrumento utilizado, si bien la mayoría de quienes respondieron la encuesta ocupa mandos medios y altos, pueden no estar en conocimiento de las acciones que desarrolla la organización o tener una impresión personal de las mismas, sesgando las respuestas. La investigación realizada tiene un diseño transversal y esto impide analizar tendencias. Un estudio longitudinal permitiría estudiar posibles patrones evolutivos e impulsores de la EC en el sector. A su vez, nos hemos concentrado solo en el sector agroalimentario; un estudio sectorial, permitiría una caracterización más profunda de la situación en el país y analizar particularidades diferenciadas. Para profundizar el estudio, se podría avanzar con un diseño cualitativo a través de casos de estudios, complementando los resultados con percepciones sobre barreras y facilitadores, key performance indicators (KPI), cómo se preparan, y cuáles se necesitan, analizando los RS publicados y que IC contienen.

\section{REFERENCIAS BIBLIOGRÁFICAS}

Barreiro-Gen, M., \& Lozano, R. (2020). How circular is the circular economy? Analysing the implementation of circular economy in organisations. Business Strategy and the Environment, 29(8), 3484-3494. https: / / doi.org/10.1002/bse.2590

Bhimani, A., Silvola, H., \& Sivabalan, P. (2016). Voluntary corporate social responsibility reporting: A study of early and late reporter motivations and outcomes. Journal of Management Accounting Research, 28(2), 77-101. https: / / doi.org/10.2308/jmar-51440

Comisión Nacional de Valores (2021). Buscador de empresas cotizadas. https: / www.cnv.gov.ar / sitioweb / empresas?seccion=buscador

Dagiliene, L., Frendzel, M., Sutiene, K., \& Wnuk-Pel, T. (2020). Wise managers think about circular economy, wiser report and analyze it. Research of environmental reporting practices in EU manufacturing companies. Journal of Cleaner Production, 274, 121968.

Del Barco Zilli, J. P., Del Barco Zilli, M. de los A., Del Barco Zilli, M. S., \& García Federici, I. (2018). Divulgación de aspectos ambientales en los informes de sostenibilidad frente al nuevo escenario internacional. Capic Review, 16, 1-19. https: / / doi.org/10.35928 / cr.vol16.2018.67

Duque-Acevedo, M., Belmonte-Ureña, L. J., Plaza-Úbeda, J. A., \& Camacho-Ferre, F. (2020). The management of agricultural waste biomass in the framework of circular economy and bioeconomy: An opportunity for greenhouse agriculture in Southeast Spain. Agronomy, 10(4), 489.

Ellen Macarthur Foundation. (2015). Towards a Circular Economy: Business Rationale for an Accelerated Transition. https: / / doi.org/2012-04-03

Ellen Macarthur Foundation. (2017). FOOD AND THE CIRCULAR ECONOMY. Learning Path. https: / / www.ellenmacarthurfoundation.org/ explore/ food-cities-the-circular-economy

Esposito, B., Sessa, M. R., Sica, D., \& Malandrino, O. (2020). Towards circular economy in the agrifood sector. A systematic literature review. In Sustainability (Switzerland) (Vol. 12, Issue 18, 


\section{p. 7401). MDPI AG. https: / / doi.org/10.3390/SU12187401}

Farooque, M., Zhang, A., \& Liu, Y. (2019). Barriers to circular food supply chains in China. Supply Chain Management, 24(5), 677-696. https:/ / doi.org/10.1108/SCM-10-2018-0345

Fattahi, F., Nookabadi, A. S., \& Kadivar, M. (2013). A model for measuring the performance of the meat supply chain. British Food Journal, 115(8), 1090-1111. https:/ / doi.org/10.1108/ BFJ-09-2011-0217

Fortunati, S., Martiniello, L., \& Morea, D. (2020). The strategic role of the corporate social responsibility and circular economy in the cosmetic industry. Sustainability (Switzerland), 12(12).

Frías-Navarro, D. (2021). Apuntes de consistencia interna de las puntuaciones de un instrumento de medida (pp. 1-22). https: / / doi.org/ https: / doi.org/10.17605/osf.io/kngtp

Geissdoerfer, M., Savaget, P., Bocken, N. M. P., \& Hultink, E. J. (2017). The Circular Economy - A new sustainability paradigm? Journal of Cleaner Production, 143, 757-768.

Ghisellini, P., Cialani, C., \& Ulgiati, S. (2016). A review on circular economy: The expected transition to a balanced interplay of environmental and economic systems. Journal of Cleaner Producti, 114, 11-32.

Gray, R., Owen, D., \& Adams, C. (2009). Some theories for social accounting?: A review essay and a tentative pedagogic categorisation of theorisations around social accounting (pp. 1-54).

Gunarathne, N., Wijayasundara, M., Senaratne, S., Kanchana, P. D. K., \& Cooray, T. (2021). Uncovering corporate disclosure for a circular economy: An analysis of sustainability and integrated reporting by Sri Lankan companies. Sustainable Production and Consumption, 27, 787-801.

Hair, J. F., Black, W. C., Babin, B., Anderson, R. E., \& Tatham, R. L. (2005). Análisis multivariante. Madrid: Prentice Hall.

Hernández Sampieri, R., \& Fernández Collado, C. (1998). Metodología de la investigación. McGraw-Hill.

Kirchherr, J., Reike, D., \& Hekkert, M. (2017). Conceptualizing the circular economy: An analysis of 114 definitions. Resources, Conservation and Recycling, 127(September), 221-232.

Kleine Jäger, J., \& Piscicelli, L. (2021). Collaborations for circular food packaging: The set-up and partner selection process. Sustainable Production and Consumption, 26, 733-740.

Laboratorio de Ecoinnovación. (2017). Sector agroalimentario. Estrategias para un mundo agrario y una industria agroalimentaria más circulares. In Colección de guías de economía circular Sector. http: / / comecyt.edomex.gob.mx/media/filer_public/65/92 / 6592b5eb-6949-47c8a402-d47b9f464a46/competitividad_sector_agroalimentario.pdf

Liu, Y., \& Bai, Y. (2014). An exploration of firms' awareness and behavior of developing circular economy: An empirical research in China. Resources, Conservation and Recycling, 87, $145-152$.

Ministerio de desarrollo productivo de la República Argentina (2021). GPS de Empresas argentinas. https: / gpsempresas.produccion.gob.ar/

Moneva, J. M., \&Llena, F. (2000). Environmental disclosures in the annual reports of large companies in Spain. European Accounting Review, 9(1), 7-29. https: / / doi.org/10.1080/096381800407923

MSCI. (2020). The global industry classification standard. https: / / www.msci.com/gics

Mura, M., Longo, M., \& Zanni, S. (2020). Circular economy in Italian SMEs: A multi-method study. Journal of Cleaner Production, 245, 118821. https: / / doi.org/10.1016/j.jclepro.2019.118821

Muscio, A., \& Sisto, R. (2020). Are agri-food systems really switching to a circular economy model? Implications for European research and innovation policy. Sustainability (Switzerland), 12(14). 
Nasution, A. H., Aula, M., \& Ardiantono, D. S. (2020). Circular economy business model design. International Journal of Integrated Supply Management, 13(2-3), 159-177.

ONU FAO. (2020). Guía práctica para la incorporación de soluciones y los desperdicios a las pérdidas de alimentos.

Pastor, S., Raffaelli, P., \& Jugon, M. (2019). Revelación de información de responsabilidad social en el sector financiero argentino. Factores explicativos. Capic Review, 17, 1-14.

Pierini \& Palmieri (2018). Importancia del sector agroindustrial en Argentina. Camara de exportaciones de la República Argentina. Recuperado de www.cera.org.ar

Platform for Accelerating the Circular Economy. (2021). Circular Economy Action Agenda - Food. February, 55.

Powell, W., \& Dimaggio, P. (1991). The new institutionalism in organizational analysis. The University of Chicago Press.

Rabasedas, M. L. (2018). Divulgación de información medioambiental: análisis exploratorio para las entidades cotizadas en Argentina. Capic Review, 16, 1-16. https:/ / doi.org/10.35928/ cr.vol16.2018.63

Reike, D., Vermeulen, W. J. V., \& Witjes, S. (2018). The circular economy: New or Refurbished as CE 3.0? - Exploring Controversies in the Conceptualization of the Circular Economy through a Focus on History and Resource Value Retention Options. Resources, Conservation and Recycling, 135(November 2017), 246-264. https: / / doi.org/10.1016/j.resconrec.2017.08.027

Rodríguez, A., Henry, G., \& Trigo, E. (2018). Latin America's developing bioeconomies : concept note for a regional panel discussion. Global Bioeconomy Summit. https:/ gbs2018.com/ home/

Salimi, N. (2021). Circular Economy in Agri-food Systems. In J. Rezaei (Ed.), Strategic Decision Making for_Sustainable Management of_Industrial Networks (8th ed., pp. 57-70). Springer.

Scarpellini, S., Marín-Vinuesa, L. M., Aranda-Usón, A., \& Portillo-Tarragona, P. (2020). Dynamic capabilities and environmental accounting for the circular economy in businesses. Sustainability Accounting, Management and Policy Journal, 11(7), 1129-1158.

Scarpellini, S., Valero-Gil, J., Moneva, J. M., \& Andreaus, M. (2020). Environmental management capabilities for a "circular eco-innovation." Business Strategy and the Environ., 29(5), 1850-1864.

Stewart, R., \& Niero, M. (2018a). Circular economy in corporate sustainability strategies: A review of corporate sustainability reports in the fast-moving consumer goods sector. Business Strategy and the Environment, 27(7), 1005-1022. https: / / doi.org/10.1002/bse.2048

Stewart, R., \& Niero, M. (2018b). Circular economy in corporate sustainability strategies: A review of corporate sustainability reports in the fast-moving consumer goods sector. Business Strategy and the Environment, 27(7), 1005-1022. https: / doi.org/10.1002/bse.2048

Sumter, D., de Koning, J., Bakker, C., \& Balkenende, R. (2021). Key competencies for design in a circular economy: Exploring gaps in design knowledge and skills for a circular economy. Sustainability (Switzerland), 13(2), 1-15. https: / / doi.org/10.3390/ su13020776

Thorne, L., Mahoney, L. S., \& Manetti, G. (2014). Motivations for issuing standalone CSR reports: a survey of Canadian firms. Accounting, Auditing \& Accountability Journal, 27(4), 686-714.

Verbeek, L. H. (2016). A Circular Economy Index for the consumer goods sector. August.

Windolph, S. E., Harms, D., \& Schaltegger, S. (2014). Motivations for Corporate Sustainability Management: Contrasting Survey Results and Implementation. Corporate Social Responsibility and Environmental Management, 21(5), 272-285. https:/ / doi.org/10.1002 / CSR.1337 
Zhu, Q., Geng, Y., \& Lai, K. hung. (2010). Circular economy practices among Chinese manufacturers varying in environmental-oriented supply chain cooperation and the performance implications. Journal of Environmental Management, 91(6), 1324-1331.

\section{ANEXO I \\ CATEGORÍAS INCLUIDAS EN CADA VARIABLE SEGÚN ENCUESTA}

\section{ESTRATEGIA DE EC}

Existe un fuerte compromiso por parte de los directivos y mandos medios con la gestión circular

Se capacita al personal y se crea conciencia sobre la estrategia de EC de la empresa

Se trabaja con programas de auditoría ambiental, como la certificación ISO 14000

Se ha diseñado una estrategia de gestión de la EC en la organización

Se cuenta con un plan de acción con metas concretas para la gestión circular del negocio

La EC es parte de los objetivos de la organización y se mide el progreso hacia esos objetivos

\section{M}

DE

\section{$2,21 \quad 1,12$}

$2,09 \quad 1,09$

$2,04 \quad 1,21$

$2,01 \quad 1,00$

$2,00 \quad 1,04$

$1,95 \quad 1,05$

\section{PRÁCTICAS DE EC}

\section{Abastecimiento y cadena de suministros}

$2,17 \quad 0,09$

El sistema de almacenamiento optimiza las condiciones de conservación de inputs y productos finales, garantizando una utilización eficiente por más tiempo

Los inputs de tipo biológico o químico utilizados son biodegradables, no tóxicos

Existen criterios de selección para proveedores y compradores industriales basados en la E.C.

Existe cooperación con empresas para establecer cadenas de suministros circulares y/o simbiosis industrial

Los inputs técnicos (excluido el packaging) provienen de materiales pre-usados o reciclados.

Producción y desarrollo de productos

$2,39 \quad 0,09$

La formulación, el diseño de productos y técnicas de producción eliminan el uso de inputs tóxicos y/o peligrosos 
Se utilizan técnicas y estándares de producción que reducen al mínimo el empleo de inputs

Las pérdidas inevitables de alimentos surgidas del proceso productivo son reelaboradas, retroalimentando las líneas de producción

Los inputs y productos son seleccionados según el destino de acuerdo a forma y tamaño

Se realizan esfuerzos e inversiones para el recupero y la reutilización de energía y el tratamiento adecuado de aguas residuales ya sea dentro de la organización o por terceros.

Se trabaja para lograr un intercambio equilibrado de nutrientes en las operaciones

La energía consumida proviene de fuentes renovables $1,68 \quad 0,91$

Comercialización y distribución

$2,18 \quad 0,09$

El packaging empleado mantiene seguros los alimentos y bebidas reduciendo los deterioros y brindando más tiempo para consumo

Los productos finales presentan diferentes tamaños o porciones

Se informa a los consumidores respecto a la forma de optimizar el uso y

almacenamiento de los alimentos y sobre la manera correcta de reutilizar o reciclar el packaging

Se trabaja con sistemas de etiquetado ecológico y / o de nutrición

El packaging utilizado está elaborado con materiales reciclados y/o

bioecológicos, reduciendo al mínimo el peso y la cantidad necesaria.

Los envases y embalajes empleados son reutilizables

Se invierte/participa en iniciativas públicas y/o privadas de EC.

Se ofrece logística inversa de empaque y / o cooperación con terceros en un sistema de depósito

Los modos de transporte utilizados son eléctricos o a base de biocombustibles

Destino final o recogida, reciclado y reutilización

$2,47 \quad 0,10$

Los subproductos / pérdidas inevitables de alimentos/residuos del proceso productivo, se utilizan en nuevos productos alimenticios o se reutilizan para alimentación animal, fertilizantes, biomateriales o medicamentos ya sea en la misma organización o en otras industrias

Los residuos no reutilizables son tratados para disminuir los efectos de su disposición final.

Se han fijado objetivos y un plan para la minimización de los residuos y las emisiones al ambiente, gaseosas y de material particulado. 
Los alimentos comestibles no destinados a la venta (por daños en el packaging, tamaño o forma inadecuada, vencimiento próximo), se redistribuyen evitando su desperdicio (Ej. Donaciones)

Los subproductos/pérdidas inevitables de alimentos/residuos se reutilizan para la generación de energía renovable (ej. biogás), que es utilizada en el proceso productivo o vendida a terceros

\section{INFORMACIÓN CIRCULAR}

Se generan informes internos sobre los resultados de la gestión circular $1,84 \quad 0,99$

Se comunican las estrategias y objetivos de EC a los grupos de interés $1,861,00$

Se comunican las prácticas y acciones de EC a los grupos de interés $1,87 \quad 1,01$

Se comunican los resultados y performance de EC a los grupos de interés $1,86 \quad 0,99$ Se utiliza información cualitativa - narrativa, textos e imágenes para comunicar sobre EC a los grupos de interés

Se utiliza información cuantitativa incluyendo indicadores específicos de EC para comunicar a los grupos de interés

Fuente: Elaboración propia en base a resultados.

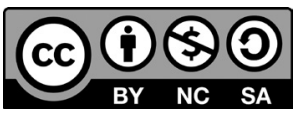

Esta obra está bajo una licencia de 